\title{
Riesgos a la salud por el uso de herbicidas
}

\author{
Xóchitl Aguilar-González ${ }^{1 /}$ Ildefonso Ronquillo-Cedillo²/ Dulce María Ávila-Nájera ${ }^{3}$ / \\ Cesáreo Rodríguez-Hernández ${ }^{4} / J o e l ~ P e d r a z a-M a n d u j a n o 5$ / Diana Laura Martínez-Jiménez ${ }^{6}$
}

DOI:

Recepción: 07/05/2021 Aceptación: 31/06/2021

\section{Resumen}

Los herbicidas son un grupo de compuestos químicos organosintéticos utilizados en la agricultura para el control de las llamadas malezas, dentro del modelo agrícola de la denominada revolución verde. Este tipo de sustancias se aplican en México, desde mediados del siglo XX, aun cuando el efecto negativo que tienen sobre el ambiente, la diversidad biológica y la salud humana se encuentra documentado.

El objetivo de esta revisión fue identificar los posibles efectos en la salud humana debido a la exposición de herbicidas. Se realizó una búsqueda de literatura en diferentes bases de datos y buscadores abiertos; se examinaron todos los artículos existentes desde el año 2010 a la fecha; sin dejar fuera los trabajos sobresalientes que aportaran información específica e importante. Asimismo, se consideraron trabajos publicados en revistas y algunos libros de especialistas en el tema.

Los resultados de las diferentes investigaciones muestran que los efectos en la salud por diversos herbicidas de uso común son varios: a corto plazo se pueden producir intoxicaciones, ya que los herbicidas se absorben en el organismo de manera directa o indirecta; mientras que a largo plazo pueden surgir daños crónicos.

Palabras clave: plaguicidas, intoxicaciones, revolución verde, riesgos de salud.

\begin{abstract}
Herbicides are a group of organosynthetic chemical compounds used in agriculture to control so-called weeds, within the agricultural model of the so-called green revolution. These types of substances have been used in Mexico since the middle of the 20th century, even when the negative effect they have on the environment, biological diversity and human health is documented.

The aim of this review was to identify possible effects on human health due to herbicide exposure. A literature search was carried out in different databases and open search engines; All existing articles from 2010 to date were examined; without leaving out the outstanding works that will provide specific and important information. Likewise, works published in magazines and some books by specialists on the subject were considered.
\end{abstract}

The results of different investigations show that the health effects of various commonly used herbicides are various: in the short term, intoxications can occur, since herbicides are absorbed into the body directly or indirectly; while long-term chronic damage can arise.

Key words: Pesticides, poisonings, green revolution, health risks.

1. Licenciada en Salud Intercultural, Universidad Intercultural del Estado de México; email: aple_pog@hotmail.com; ORCID https:// orcid.org/0000-0003-1231-9839

2. Maestro en Ciencias en Fitosanidad-Entomología y Acarología, Profesor de Tiempo Completo, Universidad Intercultural del Estado de México-División de Desarrollo Sustentable; email: ildefonso.ronquillo@uiem.edu.mx; ORCID: https://orcid.org/0000-0002-8101-3502

3. Doctora en Ciencias en Recursos Genéticos y Productividad-Ganadería, Jefa del Departamento de Investigación y Posgrado, Universidad Intercultural del Estado de México; email: dul.avna@gmail.com; ORCID: https://orcid.org/0000-0001-8535-1528

4. Doctor en Ciencias en Entomología, Profesor Investigador Titular-Colegio de Postgraduados, Campus Montecillo; email: crhernan@colpos.mx; ORCID: https://orcid.org/0000-0001-7137-3697

5. Doctor en Ciencias, Profesor de Tiempo Completo, Universidad Intercultural del Estado de México-División de Comunicación Intercultural; email: joel.pedraza@gmail.com; ORCID: https://orcid.org/0000-0003-3019-8807

6. Maestra en Ciencias Biotecnológicas, Investigadora Independiente; email: diana.martinez@uiem.edu.mx; ORCID: https://orcid. org/0000-0001-6039-7183 


\section{Introducción}

Los agroquímicos se introdujeron a México a partir de la adopción del modelo agrícola industrial en 1947. Estos fueron introducidos por la fundación Rockefeller y la Secretaría de Agricultura de México, con el objetivo de transformar a la agricultura mexicana por medio de la innovación. Este suceso fue el punto de partida para la utilización intensiva y extensiva de productos químicos sintéticos para la producción agrícola.

Esta iniciativa consistía en el cambio de un sistema tradicional a un modelo llamado revolución verde (Ceccon, 2008), cuyo objetivo fue combatir el hambre (Bisang y Campi, 2010), ya que se enfrentaba una alta demanda de alimentos y una baja producción. Por lo que se implementaron cambios en el sistema de producción, siendo uno de ellos modificar el sistema tradicional de producción agrícola por la adopción de un sistema enfocado al uso de innovaciones tecnológicas mecánicas, biológicas y químicas (Toledo, Cháires y Barón, 2002). El modelo consistía en dar nutrientes químicos exógenos (fertilizantes granulados) a la tierra, disminuir la mano de obra mediante el uso de maquinaria y controlar la maleza con herbicidas (Pichardo, 2006).

El caso del control de maleza por medio de herbicidas ha sido controversial. Por un lado, los herbicidas, considerados plaguicidas de uso agrícola, son empleados para el control de las malezas; es decir, aquellas especies vegetales que afectan los intereses del hombre al disminuir la producción y la calidad de los cultivos, debido a su gran capacidad competitiva (CEDRSSA, 2020); pero por otro, presentan efectos nocivos en la salud humana (Nivia, 2000). Además, provocan otros efectos negativos como la resistencia, presencia de residuos en las cadenas alimenticias y el ambiente físico (aire, agua, suelo) y destrucción de la fauna benéfica. Por lo tanto, el uso de estos productos ha provocado la reducción de la conectividad (interrelación trófica entre los organismos), la pérdida de biodiversidad, el desequilibrio ecológico y el decremento de la estabilidad ambiental (Badii y Landeros, 2007).

En México, el 6 de noviembre de 2019 se publicó el decreto por el que se modifica la Ley de los Impuestos Generales de Importación y de Exportación, en el que se prohibió la importación de diversas sustancias tóxicas y plaguicidas de alta peligrosidad, cuyo registro sanitario fue cancelado. De la misma manera, el pasado 31 de diciembre de 2020 se publicó un decreto en el Diario Oficial de la Federación (DOF, 2020), en el que se establecen las acciones para sustituir gradualmente el herbicida llamado glifosato, y los agroquímicos que lo contengan como ingrediente activo, por alternativas que no dañen a la salud humana y al ambiente. Esto hace necesario buscar información actualizada y veraz de los daños ocasionados por estos productos y 
sus alternativas. Es por ello que en el presente trabajo se busca recopilar información de los daños ocasionados a la salud por el uso de herbicidas, que sirva de base para tomar acciones ante dicho panorama.

\section{Desarrollo}

Se realizó una búsqueda de literatura en diferentes bases de datos y buscadores abiertos como Google Académico. Se examinaron todos los artículos existentes en el período de tiempo comprendido entre los años 2010 hasta el mes de septiembre de 2021, sin dejar fuera trabajos sobresalientes que aportaran información específica e importante. Se consideraron para la investigación trabajos publicados en revistas y algunos libros de especialistas en el tema, que trataran en específico los daños a la salud humana por los herbicidas, dejando fuera las investigaciones que se enfocaran en daños al ambiente $\mathrm{u}$ otros aspectos.

Los compuestos químicos que contienen os herbicidas provocan daños a la salud como afecciones respiratorias (Jiménez, Pantoja y Femy, 2016), dermatosis e intoxicaciones (Bejarano, 2017). Las personas se intoxican por su uso directo (por inhalación) o indirecto (absorción por vía cutánea). Se ha observado que el riesgo a la exposición de herbicidas durante su uso es alto y los químicos ingresan al organismo por diferentes vías. Esto se magnifica, ya que los productores no utilizan equipo de protección, o estos son defectuosos presentando múltiples fugas (Martínez, Ruiz, Gavilán y Mendoza, 2020).

La exposición ocupacional a formulaciones puede producir diferentes daños a la salud y dependerá del tipo de exposición y duración; cantidad o dosis de exposición y periodicidad, así como de los principios activos (Espinoza, s.f.). Sin embargo, se pueden evitar los riesgos a la salud, siguiendo lo establecido en la Norma Oficial Mexicana NOM 127-SSA, la cual regula el uso y manejo de los herbicidas (Bejarano, 2017); o en el uso de alternativas para sustituir su uso.

\section{Daños de los principales herbicidas utilizados}

La mayoría de las investigaciones recientes de herbicidas y salud humana se enfocan en glifosato y su compuesto de degradación; el ácido aminometilfosfónico (AMPA), un ácido orgánico débil con un grupo fosfonato (Duke, 2020); aunque también se encuentran investigaciones con otros herbicidas. El interés científico por el glifosato se debe a la polémica por el incremento en el uso de esta sustancia asociado a cultivos transgénicos. Desde 1996 a 2016 se incrementó hasta quince veces el cultivo de transgénicos resistentes a glifosato "Roundup Ready" (Benbrook, 2016).

A continuación, se describen los riesgos más importantes de algunos de los principales herbicidas utilizados por los campesinos en México: 
a. Paraquat o 1,1-dimetil-4,4-bipiridilo dicloruro

Es un herbicida de contacto (requiere contacto físico con la superficie del follaje), de acción rápida. Se presenta en forma líquida en concentraciones del 20\% para uso agrícola e interfiere con la fotosíntesis. Uno de formulados más comunes es el gramoxone (Viales, 2014). En México, el herbicida paraquat se encuentra registrado en diferentes formulaciones, corresponde de manera general a la categoría toxicológica III. Aunque también hay formulados en categoría II y con la nueva clasificación 1 y 4, sigue vigente su entrada a México y se siguen utilizando por los productores (COFEPRIS, 2021).

La intoxicación por paraquat, además de eventos autocidas, se da en exposiciones ocupacionales por absorción a través de la vía cutánea o conjuntiva, lo que puede causar insuficiencia orgánica múltiple, en la cual el pulmón es el órgano objetivo primario (Viales, 2014). Después de la intoxicación, la tasa de mortalidad del paciente es tan alta como 90\%, y actualmente no existe un antídoto específico. La dosis letal mínima de paraquat para humanos es de $40 \mathrm{mg} \mathrm{kg}-1$ de peso (10 $15 \mathrm{ml} \mathrm{kg-1}$ de producto concentrado al 20\%) (Peñafiel, 2019). El paraquat genera riesgos a la salud como infertilidad, en hombres que usan de manera frecuente el herbicida; en algunos casos disminuye la testosterona, ya que al ingresar este herbicida al organismo, los metabolitos residuales alteran la producción de hormonas y provocan desequilibrio (Paparella, Pavesi, Provenzal, Ombrella, y Bouvet, 2017).

\section{b. Glifosato o $\mathrm{N}$-fosfonometil-glicina}

El glifosato es un herbicida de amplio espectro, no selectivo, que elimina tanto pastos anuales y perennes como hierbas de hoja ancha (Benachour y Séralini, 2009). Los formulados contienen el surfactante polioxileno-amina, que inhibe la enzima enol-piruvil-shikimato-fosfato-sintetasa (EPSPS), evitando que las plantas elaboren tres aminoácidos aromáticos esenciales para su crecimiento y supervivencia como la fenilananina, tirosina y triptófano (Kaczewer, 2002).

La Organización Mundial de la Salud declaró al glifosato como probable cancerígeno para seres humanos; y fue clasificado en el grupo 2 (OMS, 2015), mientras que la Agencia Internacional para la Investigación sobre el Cáncer (IARC) lo clasifica como cancerígeno, catalogándolo directamente en grupo 1, (Guyton et al., 2015). Por otro lado, la Agencia de Protección Ambiental de los Estados Unidos de América considera al glifosato como un compuesto de toxicidad clase II; con toxicidad aguda dérmica y oral relativamente baja (los químicos de clase I son los de mayor toxicidad en la escala de I- IV (EPA, 2019)). 
En México, de acuerdo a la Comisión Federal para la Protección Contra Riesgos Sanitarios (COFEPRIS) $^{7}$, se consideran los formulados de glifosato dentro de la clasificación toxicológica IV; pero existen hay formulados clasificados dentro de las categorías 4 y 5 (COFEPRIS, 2021).

En cuanto a los riesgos a la salud reportados en las investigaciones, este elemento es altamente cancerígeno; acelera la muerte celular, daña el ADN y afecta la coagulación (Rico, Scoppetta, Alzate, \& González, 2016). En conjunto con otros plaguicidas, incrementa la prevalencia depresión debido a que el uso frecuente de estos altera el campo neurológico con los neurotransmisores (Lugo, 2017); puede causar toxicidad en células placentarias y del hígado, actuar como disruptor endocrino, generar afecciones respiratorias, gastrointestinales, dermatológicas y neurológicas, así como fragmentación del material genético (Salazar y Aldana, 2011). Afecta negativamente la respiración mitocondrial de los espermatozoides con su consecuencia en la infertilidad (Ferramosca et al., 2021). Asimismo, la presencia de glifosato y AMPA en orina de mujeres embarazadas se asocia con partos prematuros (Lesseur et al., 2022). Por otra parte, puede generar cáncer (Benachour y Séralini, 2009) como linfoma no Hodking, que compromete células que producen sangre (Gasnier et al., 2010). Aunque las causas son múltiples, se ha evidenciado que el glifosato ha influido para que este problema se desarrolle en el cuerpo humano con mayor frecuencia (Martínez, Reyes, Geliebter y Reyes, 2006).

Además de los daños atribuidos al glifosato directamente, el surfactante del glifosato (el polioxileno-amina) genera ulceración ocular, nausea, diarrea, eritema, inflamación, exudación y ulceración cutánea (Kaczewer, 2002).

\section{c. Atrazina o 6-cloro-N-etil-N'-(1-metile- til)- 1,3,5-triazina-2,4-diamina}

La atrazina es un herbicida selectivo que se aplica al suelo. Se absorbe por las raíces o las hojas de las hierbas y se aplica antes o después de que germine la maleza, principalmente pastos en cultivos de caña de azúcar, maíz, sorgo y trigo. Este herbicida se introdujo a la venta en 1958 (Golla, Nelms, Taylor \& Mishra, 2011). Es uno de los herbicidas más empleados a nivel mundial, ya que presenta variable velocidad de degradación biológica y ha sido detectado en cuerpos de agua superficial y subterránea (Hansen et al., 2013).

El uso de la atrazina empezó en México en 1975 (SAGARPA, 2007), por lo que existen diversas empresas que producen el herbicida en el país. Gesaprim es el nombre comercial más conocido de este herbicida, pero según la Comisión Intersecretarial para el Control del Proceso y uso de Plaguicidas, Fertilizan-

7. Dentro del documento, los autores también harán referencia a este término mediante el acrónimo. 
tes y Sustancias Toxicas (CICOPLAFEST 2005), existen otros productos con el mismo ingrediente activo. La atrazina es considerada como un "plaguicida de uso restringido", solo puede ser adquirido con licencia y su aplicación está regulada en otros países (ATSDR, 2003). Sin embargo, en México aún no se cuenta con una reglamentación de este tipo (González y González, 2021). En COFEPRIS esta sustancia se considera de uso restringido, con categoría toxicológica IV (clasificación anterior) y nivel 4 dentro de la clasificación actual; aunque existen algunas formulaciones con categoría 5 (COFEPRIS, 2021).

La exposición a la atrazina puede darse en el ambiente laboral por vía inhalatoria o dérmica. Puede producir reacciones de irritación, eritema, alteraciones en las funciones de algunos órganos; problemas de reproducción, defectos macrosómicos y alteración en los niveles de hormonas (disruptor endocrino); así como defectos de nacimiento en seres humanos (genotóxico y mutagénico) (Quintero, 2014).

\section{d. 2,4 $\mathrm{D}$ o ácido 2,4- diclorofenoxiacético}

El herbicida 2,4-D es uno de los plaguicidas sintéticos más antiguos. Salió al mercado en los años cuarenta y se convirtió, tristemente célebre, gracias a que formaba parte del compuesto químico defoliante, conocido como el "Agente naranja" en la guerra de Vietnam. El 2,4-D es, hasta el día de hoy, utilizado ampliamente alrededor del mundo (Neumeister,
2014). En México, el nombre más común es hierbamina; y se considera de uso restringido, con categoría toxicológica III y IV de la clasificación anterior. Pero, con la categorización actual, existen formulados en categoría 4 , pero también existen dentro de los niveles 3 y 5 (COFEPRIS, 2021).

El 2,4-D puede entrar al organismo generalmente por absorción a través de la piel, por inhalación o por la boca, al tragar las pequeñas gotas rociadas (en menores cantidades). Se ha reportado que al tener contacto prolongado en humanos e ingresar por vías respiratorias causa tos, ardor, mareo y pérdida temporal de coordinación muscular. Otros síntomas de envenenamiento pueden ser fatiga y debilidad con nauseas (Gallay, Mendoza y Benítez, 2007); además, se ha demostrado que tiene efectos adversos en el sistema endocrino y reproductivo. Por otro lado, como entre sus impurezas se encuentran niveles altos de dioxinas y furanos, puede tener efecto cancerígeno y mutagénico (Neumeister, 2014), tal como se ha observado en estudios epidemiológicos en los que se muestra una vinculación entre el uso de 2,4-D y algunos tipos específicos de cáncer; especialmente el sarcoma de tejido suave y el linfoma maligno (von Stackelberg, 2013).

\section{Conclusiones}

Los herbicidas son utilizados por los agricultores debido a las ventajas que otorgan en la 
producción de los diversos cultivos. No obstante, generan una serie de riesgos a la salud que van de las intoxicaciones ocupacionales hasta efectos crónicos, que se manifiestan como efectos en el sistema endocrino, problemas reproductivos e incluso cáncer de diversos tipos, que pueden ser ocasionado por el ingrediente activo de manera directa; sus productos de degradación o por los contaminantes acompañantes del producto formulado.

Esta investigación aporta información de los daños ocasionados por los herbicidas y sirve como base en la toma de decisiones informadas; así como también, es un aliciente para buscar alternativas viables y menos dañinas como las propuestas por la agroecología

\section{Referencias}

Agencia de Protección Ambiental (2019). Draft Toxicological Profile for Glyphosate. USA: Regulations.gov. https://www.regulations.gov/document/ATSDR-2019-0001-0003

Agency for Toxy Substances and Disease Regyster (2003). Reseña Toxicológica de la Atrazina. Atlanta, GA: Departamento de Salud y Servicios Humanos de EE. UU., Servicio de Salud Pública. https://www.atsdr.cdc.gov/es/toxfaqs/es_tfacts153.pdf

Badii, M. H. y Landeros, J. (2007). Plaguicidas que afectan a la salud humana y la sustentabilidad. CULCyT: Cultura Científica y Tecnológica, 4(19), 21-34.

Bejarano, F. (Ed.) (2017). Los plaguicidas altamente peligrosos en México. Texcoco, México: RAPAM.

Benachour, N. y Séralini, G. E. (2009). Glyphosate formulations induce apoptosis and necrosis in human umbilical, embryonic, and placental cells. Chemical research in toxicology, 22(1), 97-105. doi: 10.1021/tx800218n

Benbrook, C. M. (2016). Trends in glyphosate herbicide use in the United States and globally. Environmental Sciences Europe, 28(1), 1-15. doi: 10.1186/s12302-016-0070-0

Bisang, R. y Campi, M. (2010). Hambre, alta tecnología y desigualdad social: Un desafío a inicios del siglo XXI. Revista Iberoamericana de Ciencia, Tecnología y Sociedad-CTS, 5(14), 1-21.

Ceccon, E. (2008). La revolución verde: tragedia en dos actos. Ciencias, 1(091), 21-29. 
Centro de Estudios para el Desarrollo Rural Sustentable y la Soberanía Alimentaria (2020). Uso y regulación de herbicidas en México. Ciudad de México: Cámara de Diputados. http:// www.cedrssa.gob.mx/files/b/13/76Herbicidas.pdf

Comisión Federal para la Protección contra Riesgos Sanitarios (2021). Consulta de Registros Sanitarios de Plaguicidas, Nutrientes Vegetales y LMR. México. http://siipris03.cofepris.gob. $\mathrm{mx} /$ Resoluciones/Consultas/ConWebRegPlaguicida.asp

Comisión Intersecretarial para el Control del Proceso y uso de Plaguicidas, Fertilizantes y Sustancias Toxicas (2005). Catálogo Oficial de Plaguicidas. México.

Diario Oficial de la Federación (2019). Norma Oficial Mexicana NOM-127-SSA12009. Plaguicidas: que establece los requisitos del envase, embalaje y etiquetado de productos grado técnico y para uso agrícola, forestal, pecuario, jardinería, urbano, industrial y doméstico.

Duke, S. O. (2020) Glyphosate: environmental fate and impact. Weed Sci. 68, 201-207. doi: $10.1017 /$ wsc. 2019.28

Espinoza, S. B. (s.f). Glifosato. aplicaciones e impactos a la salud. Buenos Aires: Unión de docentes de la provincia de Buenos Aires (UDOCBA). http://www.udocba.org.ar/images/1313/ Informe\%20T\%C3\%A9cnico\%20Glifosato\%20-\%20SE.pdf

Ferramosca, A.; Lorenzetti, S.; Di Giacomo, M.; Murrieri, F.; Coppola, L. y Zara, V. (2021). Herbicides glyphosate and glufosinate ammonium negatively affect human sperm mitochondria respiration efficiency. Reproductive Toxicology, 99, 48-55. Doi: https://doi.org/10.1016/j.reprotox.2020.11.011

Gallay, R.; Mendoza, C. y Benítez, V. N. B. (2007). Oxidación fotocatalítica de los herbicidas 2, 4-D, Diurón y Ametrina en agua a escala de laboratorio. Santiago de Cali: Facultad de Ingeniería de la Universidad del Valle. http://biosolar.univalle.edu.co/reports/raphaelGallayDoc.pdf

Gasnier, C.; Benachour, N.; Clair, E.; Travert, C.; Langlois, F.; Laurant, C., ... y Séralini, G. E. (2010). Dig1 protects against cell death provoked by glyphosate-based herbicides in human liver cell lines. Journal of occupational medicine and toxicology, 5(1), 1-13. Doi: https:// doi.org/10.1186/1745-6673-5-29 
Golla, V.; Nelms, J.; Taylor, R. y Mishra, S. (2011). Pesticide concentrations in drinking water from farm homes: Variation between community water supply and well-water. Journal of Environmental Science and Engineering, 5(8), 955-961.

González-B., M. M. y González-B., O. M. (2021). Atrazina, un herbicida tóxico. México: Procuraduría General del Consumidor. https://www.gob.mx/profeco/es/articulos/atrazina-un-herbicida-toxico?idiom $=e s$

Guyton, K. Z.; Loomis, D.; Grosse, Y.; El Ghissassi, F., Benbrahim-Tallaa, L., Guha, N., ... y Straif, K. (2015). International Agency for Research on Cancer Monograph Working Group ILF. Carcinogenicity of tetrachlorvinphos, parathion, malathion, diazinon, and glyphosate. Lancet Oncol, 16(5), 490-491.

Hansen, A. M.; Quintanilla, L. G. T.; Pacheco, H. M.; Canela, M. V.; Márquez, L. C. G.; Garcés, R. A. G. y Antonio, A. H. (2013). Atrazina: un herbicida polémico. Revista Internacional de Contaminación Ambiental, 29, 65-84.

Jiménez, C.; Pantoja, A. y Femy, H. (2016). Riesgos en la Salud de agricultores por uso y manejo de plaguicidas, microcuenca "La Pila". Revista Univ. http://dx.doi.org/10.22267/ rus. 161803.48 .

Kaczewer, J. (2002). Toxicología del glifosato: riesgos para la salud humana. Agora Philosophica, $3,115-124$

Lesseur, C.; Pathak, K. V.; Pirrotte, P.; Martinez, M. N.; Ferguson, K. K.; Barrett, E. S., ... y Chen, J. (2022). Urinary glyphosate concentration in pregnant women in relation to length of gestation. Environmental Research, 203, 111811. Doi. https://doi.org/10.1016/j.envres.2021.111811

Lugo, L. (2017). Depresión y exposición a plaguicidas en agricultores de Opichén, Yucatán, México. México: Tesis de maestría. Centro de Investigación y de Estudios Avanzados del Instituto Politécnico Nacional, Unidad Mérida. Departamento de ecología humana. https://www. mda.cinvestav.mx/FTP/EcologiaHumana/maestria/tesis/14TesisLugoL17.pdf

Martínez A, A.; Ruiz S., L.G.; Gavilán G., A. y Mendoza C., A. (2020). Estrategia Nacional para Evitar los Riesgos al Ambiente por los Plaguicidas en México. México: Instituto Nacional de Ecología y Cambio Climático (INECC). https://www.gob.mx/cms/uploads/attachment/ file/558958/EstrategiaNacionalParaEvitarRiesgosPlaguicidas.pdf. 
Martínez, A.; Reyes, I.; Geliebter, J. y Reyes, N. (2006). Análisis toxicogenómico de potenciales alteraciones en la expresión genética en linfocitos humanos expuestos a glifosato: Implicaciones en el desarrollo de linfoma no Hodgkin. Revista de la Universidad Industrial de Santander. Salud, 38(1), 25-28.

Neumeister, L. (2014). Riesgos del herbicida 2, 4-D. publicación conjunta entre Testbiotech, U., GeneWatch, U. K., \& Network, P. A. http://www.rallt.org/boletin/boletin\%20540-600/ Bol.567\%20Riesgos\%20del\%20Herbicida\%202-4D.pdf

Nivia, E. (2000). Efectos sobre la salud y el ambiente de herbicidas que contienen glifosato. http:// www.mamacoca.org/docs_de_base/Fumigas/Nivia_Efectos_salud_ambiente_herbicidas_ con_Glifosato.pdf.

Organización Mundial de la Salud (2015). Preguntas y respuestas sobre el uso diazinón, malatión y glifosato. https://www.paho.org/hq/dmdocuments/2015/2015-cha-preg-resp-diazinon-malation-glisofato.pdf

Paparella, C.; Pavesi, A.; Provenzal, O.; Ombrella, A. y Bouvet, B. (2017). Infertilidad masculina. Exposición laboral a factores ambientales y su efecto sobre la calidad seminal. Revista Uruguaya de Medicina Interna, 2(2), 10-21.

Peñafiel, S. (2019). Intoxicación por Paraquat. Revista Ecuatoriana de Medicina EUGENIO ESPEJO, 7(11), 1-5.

Pichardo, B. (2006). La revolución verde en México. Agraria, São Paulo, 4, 40-68 https://core. ac.uk/download/pdf/268328398.pdf

Quintero-R., A. (2014). Atrazina: impacto en ecosistemas acuáticos y en la salud de la población expuesta. Memorias del Congreso Científico, 20-24 de octubre Universidad de Panamá. https://www.researchgate.net/profile/Aristides-Quintero-Rueda/publication/280125842_ Atrazina_impacto_en_ecosistemas_acuaticos_y_en_la_salud_de_la_poblacion_expuesta/links/55ab4f1108ae481aa7fbd433/Atrazina-impacto-en-ecosistemas-acuaticos-y-en-la-salud-de-la-poblacion-expuesta.pdf

Rico, D.; Scoppetta, O.; Alzate, J. P. y González F., A. (2016). Verdades científicas sobre glifosato y salud pública. Fundación Ideas para la Paz. Serie de Informes, 1, (25), 7-67 
Salazar-L., N. J. y Aldana-M., M. L. (2011). Herbicida glifosato: usos, toxicidad y regulación. BIOtecnia, 13(2), 23-28.

Secretaría de Agricultura, Ganadería, Desarrollo Rural, Pesca y Alimentación (2007). Anteproyecto de Modificación de la Norma Oficial Mexicana NOM-032-FITO-1995. http://www. cofemer-mir.gob.mx/mir/uploadtests/10046.59.59.1.NOM-\%20032-FITO-1995\%20

Toledo, V. M.; Cháires, A. y Barón, L. (2002). La modernización rural de México un análisis socioecológico. México: Secretaría del Medio Ambiente y Recursos Naturales, Instituto Nacional de Ecología y Universidad Nacional Autónoma de México.

Viales-L., G. (2014). Intoxicación por Paraquat. Medicina Legal de Costa Rica, 31(2), 88-94.

Von Stackelberg, K. (2013). A Systematic Review of Carcinogenic Outcomes and Potential Mechanisms from Exposure to 2,4-D and MCPA in the Environment. Journal of toxicology, 2013, 371610. https://doi.org/10.1155/2013/371610 